\title{
Comparison of alternative versions of the job demand-control scales in 17 European cohort studies: the IPD-Work consortium
}

Eleonor I Fransson ${ }^{1,2^{*}}$, Solja T Nyberg ${ }^{3}$, Katriina Heikkilä ${ }^{3}$, Lars Alfredsson ${ }^{1}$, De Dirk Bacquer ${ }^{4}$, G David Batty ${ }^{5,6}$, Sébastien Bonenfant ${ }^{7}$, Annalisa Casini ${ }^{8}$, Els Clays ${ }^{4}$, Marcel Goldberg ${ }^{7,9}$, France Kittel ${ }^{8}$, Markku Koskenvuo ${ }^{10}$, Anders Knutsson ${ }^{11}$, Constanze Leineweber ${ }^{12}$, Linda L Magnusson Hanson ${ }^{12}$, Maria Nordin ${ }^{13}$, Archana Singh-Manoux ${ }^{5,9}$, Sakari Suominen ${ }^{14,15}$, Jussi Vahtera ${ }^{14,16}$, Peter Westerholm ${ }^{17}$, Hugo Westerlund ${ }^{5,12}$, Marie Zins ${ }^{7,9}$, Töres Theorell ${ }^{12 \dagger}$ and Mika Kivimäki ${ }^{3,5,18+}$

\begin{abstract}
Background: Job strain (i.e., high job demands combined with low job control) is a frequently used indicator of harmful work stress, but studies have often used partial versions of the complete multi-item job demands and control scales. Understanding whether the different instruments assess the same underlying concepts has crucial implications for the interpretation of findings across studies, harmonisation of multi-cohort data for pooled analyses, and design of future studies. As part of the 'IPD-Work' (Individual-participant-data meta-analysis in working populations) consortium, we compared different versions of the demands and control scales available in 17 European cohort studies.

Methods: Six of the 17 studies had information on the complete scales and 11 on partial scales. Here, we analyse individual level data from 70751 participants of the studies which had complete scales ( 5 demand items, 6 job control items).

Results: We found high Pearson correlation coefficients between complete scales of job demands and control relative to scales with at least three items $(r>0.90)$ and for partial scales with two items only $(r=0.76-0.88)$. In comparison with scores from the complete scales, the agreement between job strain definitions was very good when only one item was missing in either the demands or the control scale (kappa > 0.80); good for job strain assessed with three demand items and all six control items (kappa > 0.68) and moderate to good when items were missing from both scales ( $\mathrm{kappa}=0.54-0.76$ ). The sensitivity was $>0.80$ when only one item was missing from either scale, decreasing when several items were missing in one or both job strain subscales.
\end{abstract}

Conclusions: Partial job demand and job control scales with at least half of the items of the complete scales, and job strain indices based on one complete and one partial scale, seemed to assess the same underlying concepts as the complete survey instruments.

Keywords: Job demands, Job control, Job strain, Work stress, Agreement

\footnotetext{
* Correspondence: eleonor.fransson@ki.se

+ Contributed equally

'Institute of Environmental Medicine, Karolinska Institutet, Box 210, SE-171 77

Stockholm, Sweden

Full list of author information is available at the end of the article
} 


\section{Background}

Work-related psychosocial factors or "stressors at work" have been linked to increased risk of ill health and mortality in some [1-8] but not all studies [9-12]. The reasons behind these inconsistencies may include differences in the socio-demographic characteristics of the study populations, variations in the stability of the work stressors during the follow-up, selection bias, and imprecise measurement, particularly of the exposure $[13,14]$. Surprisingly little attention has been paid to the fact that the operationalisation of work-related stressors have varied between cohort studies.

A frequently used measure of work stress is the twodimensional job strain model, originally described by Karasek [15] and further developed, both empirically and psychometrically, by Karasek and Theorell [16]. This model postulates that jobs characterised by a combination of high psychological demands and low control (or low decision latitude), that is, job strain, are likely to elicit work-related psychosocial stress. High psychological demands in the workplace mean that the employee has to work intensively or rapidly, and may experience conflicting expectations. Job control, in turn, refers to the degree of decision-making authority (for example, having an influence on what task to do and how to carry it out) and skill discretion (e.g., the use of personal skills on the job).

The two standardised, widely used questionnaires developed to measure the demand and control dimensions, and hence job strain, are: the Job Content Questionnaire (JCQ) [17] and the Demand Control Questionnaire (DCQ) $[18,19]$. The number of demand (five in both the JCQ and DCQ) and control items (nine in the JCQ and six in the DCQ) vary somewhat, as do the enquiries, and their response scales differ. Most of the JCQ items are expressed as statements and the respondents are asked to report if they agree or disagree with the statement on a four level Likert scale, while DCQ items are expressed as questions with the response options being frequency based (e.g., "Do you have to work very fast?" - Often, sometimes, seldom, never).

Investigators examining the relationship between job strain and health outcomes have often used partial versions of the questionnaires. Study-specific questionnaires have also been developed which can differ from the JCQ or DCQ in terms of the number of items, content and wording of the questions, and response alternatives. It is important to understand whether the different survey instruments assess the same underlying concepts, as this has implications for the interpretation of findings across studies, harmonisation of multi-cohort data for pooled analyses, and design of future studies [20].
Accordingly, the aim of the present analyses was to evaluate the comparability of alternative job demands, job control and job strain measures by assessing agreement against the complete scale. To do so, we use data from six studies together with information from additional 11 European cohort studies that comprise the "Individual-participant-data meta-analysis in working populations" (IPD-Work) Consortium.

\section{Methods}

\section{Study population}

This study is part of the "Individual-participant-data meta-analysis in working populations" (IPD-Work) consortium of European cohort studies. This collaboration was established at a workshop, the annual Four Centers Meeting in London, in November, 2008. New cohort studies have subsequently been added. The overall aim of the IPD-Work consortium is to aggregate raw data from a series of studies in order to obtain reliable estimates of the influence of psychosocial risk factors at work on chronic diseases, mental health, disability, and mortality.

In IPD-Work, a pre-defined two-stage data acquisition protocol is being used. The first stage involves the acquisition of baseline data on work stress as well as socio-demographic and lifestyle factors and the definition and harmonisation of these baseline characteristics across the studies. The second stage involves the acquisition of data on disease outcomes ascertained subsequent to the baseline survey. The present analyses were based on stage one only and were thus conducted before any linkage to disease data, planned for the second stage.

We examined the agreement between complete and partial psychological demands and control scales separately in the six cohort studies of the IPD-Work consortium that had the complete job demands and job control scales. These were: the Job Stress Study I (Belstress, Belgium) [21]; the Gaz et Electricité Cohort Study (GAZEL, France) [22]; the Health and Social Support in Finland Study (HeSSup, Finland) [23]; the Swedish Longitudinal Occupational Survey of Health (SLOSH, Sweden) $[24,25]$; the Work, Lipids and Fibrinogen Study Norrland (WOLF N, Sweden) [26]; and the Work, Lipids and Fibrinogen Study Stockholm (WOLF S, Sweden) [26]. This resulted in an analytic sample of 70751 participants. The IPD-Work cohort studies where only partial scales were available were: the Copenhagen Psychosocial Questionnaire Study, Denmark [27]; Danish Work Environment Cohort Study, Denmark [28]; Still Working study, Finland [29]; Finnish Public Sector study, Finland [30]; Heinz Nixdorf Recall Study, Germany [31]; Intervention Project on Absence and Well- 
being Study, Denmark [32]; KORA-Cooperative Health Research in the Region Augsburg/MONICA, Germany [33]; Netherlands Working Condition Survey, the Netherlands [34]; Permanent Onderzoek Leefsituatie/Continuous Survey on Living Conditions, the Netherlands [35]; PUMA-Copenhagen Burnout Inventory Study, Denmark [36]; and Whitehall II study, the United Kingdom [37]. We used the information on available job demands and control items in these 11 cohort studies to identify partial scales relevant to compare with the complete scales. The details of the design and participants in all 17 IPDWork studies have been published previously [21-37] and are briefly described in Additional file 1 .

The studies from which data was used in the present analysis were approved by the ethics committees of the University Hospital of Ghent and the Faculty of Medicine of the Université Libre de Bruxelles (Belstress); the National Commission Overseeing Ethical Data Collection in France (Commission Nationale Informatique et Liberté) (GAZEL); the Turku University Central Hospital Ethics Committee (HeSSup); the Regional Research Ethics Board in Stockholm (SLOSH); and the ethics committee at Karolinska Institutet, Stockholm (WOLF $\mathrm{N}$ and WOLF S). All studies adhered to the Helsinki Declaration on ethical principles for medical research involving human subjects. Details of ethical approval of all the IPD-Work studies are described in Additional file 1 .

\section{Definition of the complete and partial scales of job demands and control}

Complete scale measures of job demands and job control were based on five items from the psychological demands scales and six items from the control scales from the JCQ and DCQ (referred to hereafter as the "complete scales" - see table 1). This represented our referent. We omitted the three additional control items in the JCQ that did not have a corresponding item in the DCQ as a means of improving the harmonisation of the control scale across studies. We constructed partial scales based on the JCQ/DCQ items that were available in each of the IPD-work studies that did not have the complete scales. This resulted in a total of six partial demand scales; five partial control scales; and 10 partial job strain scales. The individual questionnaire items available in each study are presented in table 2 .

All studies included in the IPD-Work Consortium were designed and initiated before the IPD-Work Consortium began; the choice of instrument to measure job strain therefore varies between studies. In some studies, the wording of the job strain items differed from those in the original JCQ or DCQ, but was judged by the study coordinating authors (EF, SN, KH, TT and MiK) to sufficiently resemble the original questions such that they
Table 1 Items from the Job Content Questionnaire (JCQ) and Demand Control Questionnaire (DCQ) included in the IPD-Work

\begin{tabular}{ll}
\hline JCQ & DCQ \\
\hline Psychological demand & Psychological demand \\
\hline working very fast & work very fast \\
\hline working very hard & work very intensively \\
\hline no excessive amount of work & too much effort \\
\hline enough time & enough time \\
\hline conflicting demands & conflicting demands \\
\hline Control & Control \\
\hline learn new things & learn new things \\
\hline high level of skill & high level of skill or expertise \\
\hline require you to be creative & require you to take the initiative \\
\hline repetitive work & same thing to do over and over again \\
\hline a lot of say & deciding what you do at work \\
\hline little decision freedom & deciding how you do your work \\
\hline
\end{tabular}

could be used as proxy items. For example, the question on conflicting demands in Still Working study was expressed as "Do your superiors or workmates give you contradictory orders or instructions?" as compared with the corresponding item in the DCQ "Does your work often involve conflicting demands?"; and in POLS the item "Do you have to work under great time pressure?" was judged to capture the same content as "Do you have enough time to do everything?" in the DCQ. The scales with proxy items are labelled as "other" in table 2. Some scales were very similar to the JCQ or DCQ and only differed from them in minor aspects; they are labelled as "mainly JCQ" or "mainly DCQ" in table 2.

Using our analytical sample from the six studies with the complete scales, mean response scores for job demands items and for job control items were calculated for each study participant, for both the complete and the different partial scales. For each scale, the mean response score was calculated for participants who had answered half or more of the demand or control questions in that specific scale. However, where only two items were used in a partial scale, both items had to have non-missing values for the mean score to be calculated.

The presence of job strain was defined as having high demands (i.e., higher than the study-specific median of the demands scores) and a low control score (i.e., lower than the study-specific median of the control scores). This definition of job strain based on the quadrant approach has been widely used and will be the main method to define job strain in the IPD-Work consortium, including the present analyses. However, other approaches to derive measures of job strain from the demands and control scores have also been proposed, including the quotient method (job demands/job 
Table 2 Job demand and control items in the cohorts included in the IPD-Work meta-analysis (questionnaire used in study)*

\begin{tabular}{|c|c|c|c|c|c|c|c|c|c|c|c|c|}
\hline & $\begin{array}{c}\text { Belstress (JCQ), GAZEL } \\
\text { (JCQ), HeSSup (JCQ), } \\
\text { SLOSH (DCQ), WOLF N } \\
\text { (DCQ), WOLF S (DCQ) }\end{array}$ & $\begin{array}{l}\text { COPSOQ } \\
\text { (Mainly } \\
\text { DCQ) }\end{array}$ & $\begin{array}{l}\text { DWECS } \\
\text { (Mainly } \\
\text { DCQ) }\end{array}$ & $\begin{array}{l}\text { FPS } \\
\text { (JCQ) }\end{array}$ & $\begin{array}{l}\text { HNR } \\
\text { (JCQ) }\end{array}$ & $\begin{array}{l}\text { IPAW } \\
\text { (DCQ) }\end{array}$ & $\begin{array}{l}\text { KORA } \\
\text { (Mainly } \\
\text { JCQ) }\end{array}$ & $\begin{array}{l}\text { NWCS } \\
\text { (Other) }\end{array}$ & $\begin{array}{l}\text { POLS } \\
\text { (Other) }\end{array}$ & $\begin{array}{c}\text { PUMA } \\
\text { (Mainly } \\
\text { DCQ) }\end{array}$ & $\begin{array}{c}\text { Still } \\
\text { Working } \\
\text { (Other) }\end{array}$ & $\begin{array}{c}\text { WH II } \\
\text { (Mainly } \\
\text { DCQ) }\end{array}$ \\
\hline \multicolumn{13}{|l|}{ Job demand } \\
\hline $\begin{array}{l}\text { 1. Working } \\
\text { very fast }\end{array}$ & $x$ & $x$ & $x$ & & $x$ & $x$ & $x$ & $x$ & $x$ & $x$ & & $x$ \\
\hline $\begin{array}{l}\text { 2. Working } \\
\text { very hard/ } \\
\text { intensively }\end{array}$ & $x$ & & & $x$ & $x$ & & $x$ & $x$ & & & & $x$ \\
\hline $\begin{array}{l}\text { 3. No } \\
\text { excessive } \\
\text { amount of } \\
\text { work/too } \\
\text { much effort }\end{array}$ & $x$ & & & $x$ & $x$ & & $x$ & $x$ & & & & \\
\hline $\begin{array}{l}\text { 4. Enough } \\
\text { time }\end{array}$ & $x$ & $x$ & $x$ & $x$ & $x$ & $x$ & $x$ & $x$ & $x$ & $x$ & $x$ & $x$ \\
\hline $\begin{array}{l}\text { 5. Conflicting } \\
\text { demands }\end{array}$ & $x$ & $x$ & $x$ & & & & $x$ & & & $x$ & $x$ & $x$ \\
\hline \multicolumn{13}{|l|}{ Job control } \\
\hline $\begin{array}{l}\text { 1. Learn new } \\
\text { things }\end{array}$ & $x$ & $x$ & $x$ & $x$ & $x$ & $x$ & $x$ & & $x$ & $x$ & $x$ & $x$ \\
\hline $\begin{array}{l}\text { 2. High level } \\
\text { of skill }\end{array}$ & $x$ & $x$ & $x$ & $x$ & $x$ & $x$ & $x$ & & & $x$ & $x$ & $x$ \\
\hline $\begin{array}{l}\text { 3. Creativity/ } \\
\text { initiative }\end{array}$ & $x$ & $x$ & $x$ & $x$ & $x$ & $x$ & $x$ & $x$ & $x$ & $x$ & & $x$ \\
\hline $\begin{array}{l}\text { 4. Repetitive } \\
\text { work }\end{array}$ & $x$ & $x$ & $x$ & $x$ & $x$ & $x$ & $x$ & & $x$ & $x$ & $x$ & $x$ \\
\hline $\begin{array}{l}\text { 5. A lot of } \\
\text { say/what to } \\
\text { do }\end{array}$ & $x$ & $x$ & $x$ & $x$ & $x$ & $x$ & & & $x$ & $x$ & $x$ & $x$ \\
\hline $\begin{array}{l}\text { 6. Little } \\
\text { freedom/ } \\
\text { how to do }\end{array}$ & $x$ & $x$ & & $x$ & $x$ & $x$ & $x$ & $x$ & $x$ & & $x$ & $x$ \\
\hline
\end{tabular}

Belstress-Belgian Job Stress Study I, Belgium; COPSOQ-Copenhagen Psychosocial Questionnaire Study, Denmark; DWECS-Danish Work Environment Cohort Study, Denmark; FPS-Finnish Public Sector Study, Finland; GAZEL-Gaz et Electricité Cohort Study, France; HeSSup-Health and Social Support Study, Finland; HNR-Heinz Nixdorf Recall Study, Germany; IPAW-Intervention Project on Absence and Well-being Study, Denmark; KORA-Cooperative Health Research in the Region Augsburg/MONICA, Germany; NWCS-Netherlands Working Condition Survey, Netherland; POLS-Permanent Onderzoek Leefsituatie/Continuous Survey on Living Conditions, Netherland; PUMA-Copenhagen Burnout Inventory Study, Denmark; SLOSH-Swedish Longitudinal Occupational Survey of Health, Sweden; Still Working-Still Working study, Finland; WH II-Whitehall II study, United Kingdom; WOLF N-Work, Lipids and Fibrinogen Study, Norrland, Sweden; WOLF S-Work, Lipids and Fibrinogen Study, Stockholm, Sweden.

* JCQ = Job Content questionnaire; DCQ = Demand control questionnaire; Mainly DCQ/Mainly JCQ = Minor modifications from the original questionnaire; Other

$=$ job strain scale with proxy items. For formulations of the items used in respective study, please contact the corresponding author.

control); the logarithmic approach (log[job demands/job control]); and the subtraction approach (job demands minus job control) [38]. As a subsidiary analysis, we evaluated the agreement between the complete and partial job strain scales when applying these alternative job strain definitions, shown in Additional file 2.

\section{Statistical analysis}

The relationship between the complete and partial scales for the demands and control scales was ascertained using Pearson correlation coefficients with accompanying $95 \%$ confidence intervals. These were computed using Fisher's transformation. Sensitivity, specificity and
Kappa $(\kappa)$ statistics were calculated to evaluate the agreement between the job strain definitions based on the complete versus partial scales. The following interpretations of the Kappa statistic were utilised [39]: 0.000.20 indicates slight agreement, 0.21-0.40 fair, 0.41-0.60 moderate, 0.61-0.80 good/substantial strength of agreement, and 0.81-1.00 a very good/almost perfect agreement. All analyses were performed using SAS version 9.1 (SAS Institute Inc., Cary, NC, USA).

\section{Results}

Pearson's correlation coefficients between the complete demand scale and partial scales are shown in table 3. In 
Table 3 Correlation between the complete psychological job demands scale vs. shorter versions of the scale

\begin{tabular}{|c|c|c|c|c|c|c|}
\hline Job Demands* & $\begin{array}{c}\text { Belstress } \\
\mathrm{n}=21093\end{array}$ & $\begin{array}{c}\text { Gazel } \\
\mathrm{n}=11365\end{array}$ & $\begin{array}{c}\text { HeSSup } \\
\mathrm{n}=16784\end{array}$ & $\begin{array}{c}\text { SLOSH } \\
\mathrm{n}=10975\end{array}$ & $\begin{array}{l}\text { WOLF N } \\
n=4704\end{array}$ & $\begin{array}{c}\text { WOLF S } \\
\mathrm{n}=5670\end{array}$ \\
\hline & $r(95 \% \mathrm{Cl})^{\dagger}$ & $r(95 \% \mathrm{Cl})$ & $r(95 \% \mathrm{Cl})$ & $r(95 \% \mathrm{CI})$ & $r(95 \% \mathrm{Cl})$ & $r(95 \% \mathrm{Cl})$ \\
\hline Complete scale vs. B (4 items) & $\begin{array}{c}0.968 \\
(0.967-0.969) \\
\end{array}$ & $\begin{array}{c}0.951 \\
(0.949-0.953) \\
\end{array}$ & $\begin{array}{c}0.975 \\
(0.974-0.976) \\
\end{array}$ & $\begin{array}{c}0.972 \\
(0.970-0.973) \\
\end{array}$ & $\begin{array}{c}0.969 \\
(0.967-0.971) \\
\end{array}$ & $\begin{array}{c}0.972 \\
(0.970-0.973) \\
\end{array}$ \\
\hline Complete scale vs. C (4 items) & $\begin{array}{c}0.956 \\
(0.954-0.957) \\
\end{array}$ & $\begin{array}{c}0.943 \\
(0.941-0.945) \\
\end{array}$ & $\begin{array}{c}0.957 \\
(0.955-0.958) \\
\end{array}$ & $\begin{array}{c}0.962 \\
(0.960-0.963) \\
\end{array}$ & $\begin{array}{c}0.954 \\
(0.951-0.956) \\
\end{array}$ & $\begin{array}{c}0.961 \\
(0.959-0.963) \\
\end{array}$ \\
\hline Complete scale vs. D (3 items) & $\begin{array}{c}0.928 \\
(0.927-0.930)\end{array}$ & $\begin{array}{c}0.903 \\
(0.900-0.907)\end{array}$ & $\begin{array}{c}0.932 \\
(0.930-0.934)\end{array}$ & $\begin{array}{c}0.933 \\
(0.931-0.935)\end{array}$ & $\begin{array}{c}0.927 \\
(0.923-0.931)\end{array}$ & $\begin{array}{c}0.933 \\
(0.929-0.936)\end{array}$ \\
\hline Complete scale vs. E (3 items) & $\begin{array}{c}0.928 \\
(0.926-0.930)\end{array}$ & $\begin{array}{c}0.902 \\
(0.899-0.906)\end{array}$ & $\begin{array}{c}0.924 \\
(0.921-0.926)\end{array}$ & $\begin{array}{c}0.914 \\
(0.911-0.917)\end{array}$ & $\begin{array}{c}0.914 \\
(0.909-0.919) \\
\end{array}$ & $\begin{array}{c}0.912 \\
(0.907-0.916)\end{array}$ \\
\hline Complete scale vs. F (2 items) & $\begin{array}{c}0.876 \\
(0.872-0.879)\end{array}$ & $\begin{array}{c}0.854 \\
(0.848-0.858)\end{array}$ & $\begin{array}{c}0.836 \\
(0.831-0.840)\end{array}$ & $\begin{array}{c}0.855 \\
(0.850-0.860)\end{array}$ & $\begin{array}{c}0.837 \\
(0.828-0.845)\end{array}$ & $\begin{array}{c}0.844 \\
(0.836-0.851)\end{array}$ \\
\hline Complete scale vs. G (2 items) & $\begin{array}{c}0.782 \\
(0.776-0.787)\end{array}$ & $\begin{array}{c}0.759 \\
(0.751-0.767)\end{array}$ & $\begin{array}{c}0.820 \\
(0.815-0.825)\end{array}$ & $\begin{array}{c}0.805 \\
(0.799-0.812)\end{array}$ & $\begin{array}{c}0.808 \\
(0.798-0.818)\end{array}$ & $\begin{array}{c}0.817 \\
(0.808-0.825)\end{array}$ \\
\hline
\end{tabular}

*Abbreviated items of the complete scale: 1. "Work very fast"; 2. "Work very hard/intensively"; 3. " No excessive work/Too much effort "; 4 . "Enough time"; 5. "Conflicting demands".

Version $B$ include items 1, 2, 4, 5; version $C$ items 1, 2, 3, 4; version $D$ items 2, 3, 4; version $E$ items 1, 4, 5; version $F$ items 1 , 4; and version $G$ items 4 , 5 .

${ }^{\dagger}$ Pearson product-moment correlation coefficient $(r)$ and $95 \%$ confidence interval $(95 \% \mathrm{Cl})$.

all six studies included in the present analysis, the correlation coefficient was $r>0.94$ for partial scales comprising four items, and $r>0.90$ when the partial scale was based on three items. For partial scales with only two items, the correlation coefficient was somewhat lower ( $\mathrm{r}$ $=0.76$ to 0.88$)$, depending on the cohort and item content.

Table 4 shows that a largely similar pattern of correlations was observed for the control scale. The correlation coefficients between the complete scale with six items and the partial scales comprising five items were very high $(r \geq 0.96)$, whereas the relationship between the complete scale and partial scales with two items were slightly lower in magnitude $(\mathrm{r}=0.81$ to 0.87$)$.

Table 5 presents the sensitivity, specificity and Kappa statistics comparing the job strain definition based on complete and partial job demands and control scales. There was a consistent pattern across the studies, with the agreement between job strain definitions based on complete and partial scales being very good $(k>0.80)$, and sensitivity $>0.80$ in 14 of 18 analyses, when only one item of either job demands scale or job control scale was missing. When the job strain definition was based on three demand items and all six control items, the agreement varied between good and very good $(\kappa>$ 0.68 ). This was also seen, with one exception, for job strain definitions based on only two demand items but all six control items. When one or more items were missing in both the demands and control scales, most Kappa statistics $(\mathrm{n}=18 / 24)$ indicated at least good agreement $(\kappa>0.60)$, although for some comparisons (n $=6 / 24)$ the agreement was moderate $(\kappa=0.54$ to 0.60$)$.

Table 4 Correlation between the complete job control scale vs. shorter versions of the scale

\begin{tabular}{|c|c|c|c|c|c|c|}
\hline Job control* & $\begin{array}{c}\text { Belstress } \\
n=21160\end{array}$ & $\begin{array}{c}\text { Gazel } \\
\mathrm{n}=11389\end{array}$ & $\begin{array}{c}\text { HeSSup } \\
n=16833\end{array}$ & $\begin{array}{c}\text { SLOSH } \\
\mathrm{n}=10981\end{array}$ & $\begin{array}{l}\text { WOLF N } \\
n=4707\end{array}$ & $\begin{array}{l}\text { WOLF S } \\
\mathrm{n}=5681\end{array}$ \\
\hline & $r(95 \% \mathrm{Cl})^{\dagger}$ & $r(95 \% \mathrm{Cl})$ & $r(95 \% \mathrm{Cl})$ & $r(95 \% \mathrm{Cl})$ & $r(95 \% \mathrm{Cl})$ & $r(95 \% \mathrm{Cl})$ \\
\hline Complete scale vs. B (5 items) & $\begin{array}{c}0.975 \\
(0.975-0.976)\end{array}$ & $\begin{array}{c}0.967 \\
(0.966-0.969)\end{array}$ & $\begin{array}{c}0.975 \\
(0.974-0.975)\end{array}$ & $\begin{array}{c}0.977 \\
(0.976-0.977)\end{array}$ & $\begin{array}{c}0.976 \\
(0.975-0.978)\end{array}$ & $\begin{array}{c}0.983 \\
(0.982-0.984)\end{array}$ \\
\hline Complete scale vs. C (5 items) & $\begin{array}{c}0.974 \\
(0.974-0.975)\end{array}$ & $\begin{array}{c}0.973 \\
(0.972-0.974)\end{array}$ & $\begin{array}{c}0.975 \\
(0.975-0.976)\end{array}$ & $\begin{array}{c}0.959 \\
(0.957-0.960)\end{array}$ & $\begin{array}{c}0.957 \\
(0.954-0.959) \\
\end{array}$ & $\begin{array}{c}0.971 \\
(0.969-0.972)\end{array}$ \\
\hline Complete scale vs. D (5 items) & $\begin{array}{c}0.974 \\
(0.973-0.975) \\
\end{array}$ & $\begin{array}{c}0.970 \\
(0.969-0.971) \\
\end{array}$ & $\begin{array}{c}0.979 \\
(0.978-0.979) \\
\end{array}$ & $\begin{array}{c}0.981 \\
(0.980-0.982) \\
\end{array}$ & $\begin{array}{c}0.977 \\
(0.975-0.978) \\
\end{array}$ & $\begin{array}{c}0.984 \\
(0.984-0.985) \\
\end{array}$ \\
\hline Complete scale vs. E (5 items) & $\begin{array}{c}0.967 \\
(0.966-0.968)\end{array}$ & $\begin{array}{c}0.960 \\
(0.958-0.961)\end{array}$ & $\begin{array}{c}0.972 \\
(0.971-0.973)\end{array}$ & $\begin{array}{c}0.960 \\
(0.958-0.961)\end{array}$ & $\begin{array}{c}0.968 \\
(0.966-0.970) \\
\end{array}$ & $\begin{array}{c}0.978 \\
(0.977-0.979) \\
\end{array}$ \\
\hline Complete scale vs. F (2 items) & $\begin{array}{c}0.839 \\
(0.835-0.843)\end{array}$ & $\begin{array}{c}0.826 \\
(0.820-0.831)\end{array}$ & $\begin{array}{c}0.860 \\
(0.856-0.863)\end{array}$ & $\begin{array}{c}0.814 \\
(0.808-0.821)\end{array}$ & $\begin{array}{c}0.840 \\
(0.831-0.848)\end{array}$ & $\begin{array}{c}0.866 \\
(0.860-0.873)\end{array}$ \\
\hline
\end{tabular}

*Abbreviated items of the complete scale: 1. "Learn new things"; 2. "High level of skill"; 3. "Require creativity/initiative"; 4. "Repetitive work"; 5. "A lot of say"/ "Deciding what to do"; 6. "Deciding how".

Version B include items: 1,2,4,5,6; version C items: 1,2,3,4,6; version D items:1,3,4,5,6; version $\mathrm{E}$ items: 1,2,3,4,5; and version $\mathrm{F}$ items: 3,6 .

${ }^{\dagger}$ Pearson product-moment correlation coefficient $(r)$ and $95 \%$ confidence interval $(95 \% \mathrm{Cl})$. 
Table 5 The agreement between job strain definitions using the complete vs. partial scales

\begin{tabular}{|c|c|c|c|c|c|c|c|c|c|c|c|c|}
\hline Job strain & $\begin{array}{c}\text { Belstress } \\
N= \\
21024\end{array}$ & & $\begin{array}{c}\text { Gazel } \\
N= \\
11362 \\
\end{array}$ & & $\begin{array}{c}\text { HeSSup } \\
N= \\
16773\end{array}$ & & $\begin{array}{c}\text { SLOSH } \\
N= \\
10970\end{array}$ & & $\begin{array}{l}\text { WOLF N } \\
N=4702\end{array}$ & & $\begin{array}{c}\text { WOLF S } \\
N=5667\end{array}$ & \\
\hline Version of partial scales* & $\begin{array}{l}\text { Sensitivity } \\
\text { Specificity }\end{array}$ & $\kappa^{\dagger}$ & $\begin{array}{l}\text { Sensitivity } \\
\text { Specificity }\end{array}$ & $\kappa$ & $\begin{array}{l}\text { Sensitivity } \\
\text { Specificity }\end{array}$ & $\kappa$ & $\begin{array}{l}\text { Sensitivity } \\
\text { Specificity }\end{array}$ & $\kappa$ & $\begin{array}{l}\text { Sensitivity } \\
\text { Specificity }\end{array}$ & $\kappa$ & $\begin{array}{l}\text { Sensitivity } \\
\text { Specificity }\end{array}$ & $\kappa$ \\
\hline \multicolumn{13}{|c|}{ Complete demands and control scale vs. complete demands and partial control scale } \\
\hline $\begin{array}{l}\text { Demands version } A \text {, control version C } \\
\text { (5 items) }\end{array}$ & $\begin{array}{l}0.85 \\
1.00\end{array}$ & 0.90 & $\begin{array}{l}0.87 \\
0.99\end{array}$ & 0.90 & $\begin{array}{l}0.86 \\
0.99\end{array}$ & 0.88 & $\begin{array}{l}0.92 \\
0.97\end{array}$ & 0.88 & $\begin{array}{l}0.97 \\
0.95\end{array}$ & 0.81 & $\begin{array}{l}0.89 \\
0.99\end{array}$ & 0.89 \\
\hline \multicolumn{13}{|c|}{ Complete demands and control scale vs. partial demands and complete control scale } \\
\hline $\begin{array}{l}\text { Demands version B (4 items), control } \\
\text { version A }\end{array}$ & $\begin{array}{l}0.83 \\
1.00\end{array}$ & 0.88 & $\begin{array}{l}0.77 \\
1.00\end{array}$ & 0.85 & $\begin{array}{l}0.93 \\
0.98\end{array}$ & 0.91 & $\begin{array}{l}0.97 \\
0.98\end{array}$ & 0.93 & $\begin{array}{l}0.74 \\
1.00\end{array}$ & 0.83 & $\begin{array}{l}0.98 \\
0.96\end{array}$ & 0.87 \\
\hline $\begin{array}{l}\text { Demands version C (4 items), control } \\
\text { version A }\end{array}$ & $\begin{array}{l}0.83 \\
1.00\end{array}$ & 0.87 & $\begin{array}{l}0.85 \\
0.99\end{array}$ & 0.87 & $\begin{array}{l}0.93 \\
0.97\end{array}$ & 0.87 & $\begin{array}{l}0.75 \\
1.00\end{array}$ & 0.82 & $\begin{array}{l}0.79 \\
0.99\end{array}$ & 0.85 & $\begin{array}{l}0.96 \\
0.96\end{array}$ & 0.86 \\
\hline $\begin{array}{l}\text { Demands version D (3 items), control } \\
\text { version A }\end{array}$ & $\begin{array}{l}0.84 \\
0.99\end{array}$ & 0.86 & $\begin{array}{l}0.56 \\
1.00\end{array}$ & 0.68 & $\begin{array}{l}0.88 \\
0.97\end{array}$ & 0.85 & $\begin{array}{l}0.62 \\
1.00\end{array}$ & 0.72 & $\begin{array}{l}0.72 \\
0.99\end{array}$ & 0.79 & $\begin{array}{l}0.92 \\
0.96\end{array}$ & 0.84 \\
\hline $\begin{array}{l}\text { Demands version E (3 items), control } \\
\text { version } A\end{array}$ & $\begin{array}{l}0.90 \\
0.97\end{array}$ & 0.86 & $\begin{array}{l}0.81 \\
0.99\end{array}$ & 0.84 & $\begin{array}{l}0.91 \\
0.95\end{array}$ & 0.83 & $\begin{array}{l}0.62 \\
1.00\end{array}$ & 0.71 & $\begin{array}{l}0.76 \\
0.99\end{array}$ & 0.80 & $\begin{array}{l}0.61 \\
0.99\end{array}$ & 0.69 \\
\hline $\begin{array}{l}\text { Demands version F (2 items), control } \\
\text { version A }\end{array}$ & $\begin{array}{l}0.70 \\
0.99\end{array}$ & 0.77 & $\begin{array}{l}0.66 \\
0.99\end{array}$ & 0.74 & $\begin{array}{l}0.66 \\
0.98\end{array}$ & 0.70 & $\begin{array}{l}0.69 \\
0.98\end{array}$ & 0.75 & $\begin{array}{l}0.46 \\
0.99\end{array}$ & 0.58 & $\begin{array}{l}0.65 \\
0.97\end{array}$ & 0.68 \\
\hline \multicolumn{13}{|c|}{ Complete demands and control scale vs. partial demands and partial control scale } \\
\hline $\begin{array}{l}\text { Demands version C (4 items), control } \\
\text { version F ( } 2 \text { items) }\end{array}$ & $\begin{array}{l}0.67 \\
0.96\end{array}$ & 0.67 & $\begin{array}{l}0.66 \\
0.96\end{array}$ & 0.64 & $\begin{array}{l}0.78 \\
0.93\end{array}$ & 0.68 & $\begin{array}{l}0.58 \\
0.97\end{array}$ & 0.62 & $\begin{array}{l}0.68 \\
0.93\end{array}$ & 0.58 & $\begin{array}{l}0.63 \\
0.95\end{array}$ & 0.62 \\
\hline $\begin{array}{l}\text { Demands version E ( } 3 \text { items), control } \\
\text { version } E \text { ( } 5 \text { items) }\end{array}$ & $\begin{array}{l}0.75 \\
0.97\end{array}$ & 0.76 & $\begin{array}{l}0.66 \\
0.99\end{array}$ & 0.72 & $\begin{array}{l}0.78 \\
0.95\end{array}$ & 0.73 & $\begin{array}{l}0.57 \\
0.98\end{array}$ & 0.64 & $\begin{array}{l}0.72 \\
0.97\end{array}$ & 0.72 & $\begin{array}{l}0.45 \\
0.99\end{array}$ & 0.55 \\
\hline $\begin{array}{l}\text { Demands version F ( } 2 \text { items), control } \\
\text { version D ( } 5 \text { items) }\end{array}$ & $\begin{array}{l}0.65 \\
0.98 \\
\end{array}$ & 0.71 & $\begin{array}{l}0.66 \\
0.93 \\
\end{array}$ & 0.57 & $\begin{array}{l}0.62 \\
0.97 \\
\end{array}$ & 0.64 & $\begin{array}{l}0.63 \\
0.99 \\
\end{array}$ & 0.70 & $\begin{array}{l}0.45 \\
0.99 \\
\end{array}$ & 0.54 & $\begin{array}{l}0.57 \\
0.98 \\
\end{array}$ & 0.62 \\
\hline $\begin{array}{l}\text { Demands version G (2 items), control } \\
\text { version B ( } 5 \text { items) }\end{array}$ & $\begin{array}{l}0.47 \\
0.98\end{array}$ & 0.55 & $\begin{array}{l}0.72 \\
0.95\end{array}$ & 0.66 & $\begin{array}{l}0.73 \\
0.94\end{array}$ & 0.67 & $\begin{array}{l}0.70 \\
0.96\end{array}$ & 0.70 & $\begin{array}{l}0.62 \\
0.97\end{array}$ & 0.64 & $\begin{array}{l}0.43 \\
0.99\end{array}$ & 0.54 \\
\hline
\end{tabular}

*Abbreviated items of the complete demands scale (version A): 1. "Work very fast"; 2. "Work very hard/intensively"; 3 . "Too much effort/No excessive work"; 4. "Enough time"; 5. "Conflicting demands". Version B include items 1, 2, 4, 5; version $C$ items 1, 2, 3, 4; version D items 2, 3, 4; version E items 1, 4, 5; version $\mathrm{F}$ items 1, 4; and version $\mathrm{G}$ items 4, 5.

Abbreviated items of the complete control scale (version A): 1. "Learn new things"; 2. "High level of skill"; 3. "Require creativity/initiative"; 4. "Repetitive work"; 5. "A lot of say"/"Deciding what to do"; 6. "Deciding how". Version B include items: 1,2,4,5,6; version C items: 1,2,3,4,6; version D items:1,3,4,5,6; version E items:

1,2,3,4,5; and version $\mathrm{F}$ items: 3,6.

${ }^{\dagger}$ Kappa statistic $(\kappa)$

As expected, the sensitivity decreased when several items were missing in one or both scales. The subsidiary analyses using alternative methods to define job strain yielded a similar pattern of results as the main analysis defining job strain by the quadrant approach (Additional file 2 , tables 4 and 5).

\section{Discussion}

The aim of the present analyses was to evaluate the comparability of alternative job demands, job control and job strain measures by assessing agreement against the complete scales. To do so, we analysed data from a total of 70751 participants in six European cohort studies with complete data on five job demand and six control items. We found very high correlation coefficients between the complete and partial job demands and control scales, which included a minimum of three items. The agreement for the dichotomised job strain measure was 'good' to 'very good' when at least one of the underlying subscales was complete. When one or more of the items of the underlying scales were excluded, this agreement ranged from moderate to good, depending on the specific items left out in the partial versions.

\section{Strengths and weaknesses}

The main strength of the present study is the utilisation of data from multiple independent cohort studies which collectively comprised a very large analytical sample, so providing a high level of statistical precision. Despite slight variation in the exact wording of the questionnaire items between these studies, our findings were remarkably consistent across the six cohort studies drawn from Belgium, France, Finland and Sweden. This supports the generalisability of the present analysis across different settings in four European countries. However, in the studies included in the IPD-Work Consortium, no standardised procedure had been followed to translate the original job strain questionnaire across all studies. Thus, cross-cultural adaptation of the job strain instrument 
remains a source of error for an individual-participant data meta-analysis project on job strain. Based on a review of job demand and control questions used in 17 European cohort studies, our tests were limited to a total of 10 different partial scales that were available in these studies. It is therefore unclear how other scale modifications, including those with additional study-specific questions, agree with the complete scales. These differences, as well as those related to translation and cultural meaning of the wording, may affect the assessment of demands and control in international comparative studies [40].

\section{Comparison with previous studies}

Previously, a comparison of JCQ and DCQ-like questionnaires has been conducted in 682 participants in the JACE study [20]. The investigators in that study found a moderate agreement between median-based job strain classification using a 14-item JCQ (five demand items, nine control items) compared with the 11-item DCQ (five demand items, six control items). Attempts were made to increase the comparability between the scales by developing comparability-facilitating algorithms, as well as using regression models, in order to convert the DCQ scores to the same scale as the JCQ scores. However, with regard to the median based job strain classification, these transformations did not meaningfully improve the agreement between the JCQ and DCQ [20]. We chose a different approach to harmonise the JCQ and DCQ scales, and used the five and six comparable items in the JCQ and DCQ as the "complete" scales. These provided us a reference measurement to examine the validity of partial versions available in the existing cohort studies.

\section{Implications}

In epidemiological studies, researchers often have to make a trade-off between the amount of data they would like to collect and the amount of data it is possible to collect without increasing the non-response due to overly burdensome inventories. In these circumstances, it is not uncommon for the original scales to be abbreviated. Research using job strain as the exposure measure has not always been based on validated standardised protocols and modifications in the questionnaire may have contributed to inconsistencies in the observed job strain-disease association across various studies. For example, in a recent meta-analysis of ten prospective cohort studies, the authors reported a pooled relative risk of 1.4 (95\% confidence intervals 1.11.8) for incident coronary heart disease among participants with job strain compared to those without [41]. However, there was significant heterogeneity among datasets, with effect estimates close to the null value, or even opposite to the expected direction, in some studies [41]. Even though our study showed reasonable agreement for all the investigated partial scales with the complete scale, the agreement as well as the sensitivity decreased when several items were missing in the two sub scales of the job strain measure. Lower sensitivity implies increased risk of misclassification of job strain when using abbreviated scales, which may attenuate or inflate a true relationship between job strain and an outcome of interest. When using abbreviated scales the item content is important to consider. The control scale in the job strain index is composed of two dimensions -skill discretion and decision authority- and both these dimensions should be covered in a partial scale to measure the same construct as the complete JCQ/DCQ scale. This was the case in all the 17 studies comprising IPD-Work.

\section{Conclusions}

Information on the agreement between alternative operationalisations of job strain may help with the interpretation of previous findings, and harmonisation of multicohort data for pooled analyses. Our study provides information on the agreement between complete and partial job strain scales based on existing data from several European cohort studies. A high agreement for partial scales with at least half of the items of the complete scales, and an accurate classification of job strain when at least one of the scales has no missing items, suggest that these abbreviated scales assess the same underlying concepts as the complete survey instrument. However, all the partial scales in the present study (including the subscales comprising only two items), showed high to reasonable agreement with the complete scales. In order to capture the theoretical background for job strain and to measure the same construct as the complete JCQ/ DCQ, it is important that the abbreviated control scales cover both the skill discretion and the decision authority dimensions.

\section{Additional material}

Additional file 1: Description of the studies included in IPD-Work Consortium

Additional file 2: Appendix tables 1, 2, 3, 4, 5, correlations, sensitivity, specificity and Kappa statistics for complete and partial job strain scales defined by the quotient, logarithmic, and subtraction approaches.

\section{Acknowledgements}

The authors would like to thank Tuula Oksanen, Jaana Pentti, Paula Salo, Marianna Virtanen, (Finnish Public Sector study), Nico Dragano, Thorsten Lunau, Johannes Siegrist, Raimund Erbel, Karl-Heinz Jöckel (Heinz Nixdorf Recall (HNR) study), Ida E.H. Madsen, Reiner Rugulies, Hermann Burr, 
Marianne Borritz, Martin L. Nielsen (Copenhagen Psychosocial Questionnaire (COPSOQ); Danish Work Environment Cohort Study (DWECS); Intervention Project on Absence and Well-being (IPAW); and Burnout, Motivation and Job Satisfaction (PUMA) studies), Jane E. Ferrie, Michael G. Marmot (Whitehall II study), Goedele A. Geuskens, Wendela E. Hooftman, Irene L. Houtman (Permanent Onderzoek Leefsituatie (POLS); Netherlands Working Conditions Survey (NWCS)), Matti Joensuu, Aki Koskinen, Anne Kouvonen, Ari Väänänen (Still Working study), for IPD-Work Consortium collaboration and providing information on job strain items available in the respective studies. The IPD work consortium is supported by the EU New OSH ERA research programme (funded by the Finnish Work Environment Fund, Finland, the Swedish Council for Working Life and Social Research, Sweden (2009-2126), the German Social Accident Insurance, Germany, the Danish National Research Centre for the Working Environment), the Academy of Finland (grant \#132944), the BUPA Foundation (grant \#22094477) and the Ministry of Social Affairs and Employment, the Netherlands. EIF is supported by the Swedish council for working life and social research (2009-2126, 2010-1823). STN and KH are funded by the Academy of Finland and the Finnish Work Environment Foundation. LA is funded by the Swedish Council for Working Life and Social Research (2007-0740). DdB, AC, EC, FK and the Belstress studies are supported by the Belgian Federal Science Policy Office, the National Research Foundation (FWO/FNRS) and the Belgian Federal Public Service of Employment, Labour and Social Dialogue and the European Social Fund. GDB is a Wellcome Trust Fellow. CL is funded by the Swedish Council for Working Life and Social Research (2005-0734) and the Swedish Research Council (825-2009-6192). LLMH is supported by the Swedish Council for Working Life and Social Research (2008-1077). ASM is supported by a "European Young Investigator Award" from the European Science Foundation and the National Institute of Aging, NIH (R01AG013196 (PI); R01AG034454) (PI)). HW is financed by a research program grant from the Swedish Council for Working Life and Social Research (2007-1143). MiK is funded by the Academy of Finland, the UK BUPA Foundation, the Medical Research Council, UK, and the US National Institutes of Health (R01HL036310; R01AG034454). The funders of the consortium had no role in the study design, data collection and analysis or writing of the manuscript, or in the decision to submit the manuscript for publication.

\section{Author details}

'Institute of Environmental Medicine, Karolinska Institutet, Box 210, SE-171 77 Stockholm, Sweden. ${ }^{2}$ School of Health Sciences, Jönköping University, Jönköping, Sweden. ${ }^{3}$ Finnish Institute of Occupational Health, Helsinki, Finland. ${ }^{4}$ Department of Public Health, Ghent University, Ghent, Belgium. ${ }^{5}$ Department of Epidemiology and Public Health, University College London, London, UK. ${ }^{6}$ MRC Centre for Cognitive Ageing and Cognitive Epidemiology, University of Edinburgh, Edinburgh, UK. ' Versailles-Saint Quentin University, Versailles, France. ${ }^{8}$ School of Public Health, Université Libre de Bruxelles, Brussels, Belgium. ${ }^{9}$ Inserm U1018, Centre for Research in Epidemiology and Population Health, Villejuif, France. ${ }^{10}$ Department of Public Health, University of Helsinki, Helsinki, Finland. ${ }^{11}$ Department of Health Sciences, Mid Sweden University, Sundsvall, Sweden. ${ }^{12}$ Stress Research Institute, Stockholm University, Stockholm, Sweden. ${ }^{13}$ Department of Public Health and Clinical Medicine, Occupational and Environmental Medicine, Umeå University, Umeå, Sweden. ${ }^{14}$ Department of Public Health, University of Turku, Turku, Finland. ${ }^{15}$ Folkhälsan Research Center, Helsinki, Finland. ${ }^{16}$ Finnish Institute of Occupational Health, Turku, Finland. ${ }^{17}$ Occupational and Environmental Medicine, Uppsala University, Uppsala, Sweden. ${ }^{18}$ Department of Behavioral Sciences, University of Helsinki, Helsinki, Finland.

\section{Authors' contributions}

All authors participated in designing the study, generating hypotheses, interpreting the data and writing or critically reviewing the paper. Eleonor I Fransson, Töres Theorell and Mika Kivimäki analysed the data and wrote the first draft. All authors read and approved the final manuscript.

\section{Competing interests}

The authors declare that they have no competing interests.

Received: 15 July 2011 Accepted: 20 January 2012

Published: 20 January 2012

\section{References}

1. de Jonge J, van Vegchel N, Shimazu A, Schaufeli W, Dormann C: A longitudinal test of the demand-control model using specific job demands and specific job control. Int J Behav Med 2010, 17(2):125-133.

2. Heraclides A, Chandola T, Witte DR, Brunner EJ: Psychosocial stress at work doubles the risk of type 2 diabetes in middle-aged women: evidence from the Whitehall II study. Diabetes Care 2009, 32(12):2230-2235.

3. Jansson C, Jeding K, Lagergren J: Job strain and risk of esophageal and cardia cancers. Cancer Epidemiol 2009, 33(6):473-475.

4. Kivimaki M, Leino-Arjas P, Luukkonen R, Riihimaki H, Vahtera J, Kirjonen J: Work stress and risk of cardiovascular mortality: prospective cohort study of industrial employees. BMJ 2002, 325(7369):857.

5. Laszlo KD, Ahnve S, Hallqvist J, Ahlbom A, Janszky l: Job strain predicts recurrent events after a first acute myocardial infarction: the Stockholm Heart Epidemiology Program. J Intern Med 2010, 267(6):599-611.

6. Lindeberg SI, Rosvall M, Choi B, Canivet C, Isacsson SO, Karasek R, Ostergren PO: Psychosocial working conditions and exhaustion in a working population sample of Swedish middle-aged men and women. Eur J Public Health 2011, 21(2):190-196.

7. Netterstrom B, Kristensen TS, Sjol A: Psychological job demands increase the risk of ischaemic heart disease: a 14-year cohort study of employed Danish men. Eur J Cardiovasc Prev Rehabil 2006, 13(3):414-420.

8. Tsutsumi A, Kayaba K, Kario K, Ishikawa S: Prospective study on occupational stress and risk of stroke. Arch Intern Med 2009, 169(1):56-61.

9. Eaker ED, Sullivan LM, Kelly-Hayes M, D'Agostino RB Sr, Benjamin EJ: Does job strain increase the risk for coronary heart disease or death in men and women? The Framingham Offspring Study. Am J Epidemiol 2004, 159(10):950-958.

10. Greenlund KJ, Kiefe Cl, Giles WH, Liu K: Associations of job strain and occupation with subclinical atherosclerosis: The CARDIA Study. Ann Epidemiol 2010, 20(5):323-331.

11. Kuper $\mathrm{H}$, Adami HO, Theorell T, Weiderpass E: The socioeconomic gradient in the incidence of stroke: a prospective study in middle-aged women in Sweden. Stroke 2007, 38(1):27-33.

12. Lee $S$, Colditz G, Berkman L, Kawachi I: A prospective study of job strain and coronary heart disease in US women. Int J Epidemiol 2002, 31(6):1147-1153, discussion 1154.

13. Kivimaki M, Head J, Ferrie JE, Brunner E, Marmot MG, Vahtera J, Shipley MJ: Why is evidence on job strain and coronary heart disease mixed? An illustration of measurement challenges in the Whitehall II study. Psychosom Med 2006, 68(3):398-401.

14. Kivimaki $M$, Vahtera J, Elovainio $M$, Keltikangas-Jarvinen L, Virtanen $M$, Hintsanen M, Vaananen A, Singh-Manoux A, Ferrie JE: What are the next steps for research on work stress and coronary heart disease? SJWEH Supp/ 2008, 6:33-40.

15. Karasek R: Job demands, job decision latitude, and mental strain: Implications for job redesign. Adm Sci Q 1979, 24:285-308.

16. Karasek R, Theorell T: Healthy Work: Stress, productivity and the reconstruction of working life New York: Basic Books, Inc; 1990.

17. Job Content Questionnaire, Recommended format. [http://www.jcqcenter. org/].

18. Theorell $\mathrm{T}$ : The demand-control-support model for studying health in relation to the work environment - an interactive model. In Behavioral medicine approaches to cardiovascular disease prevention. Edited by: OrthGomér K, Schneiderman N. Mahwah, NJ: Lawrence Erlbaum Associates; 1996:69-85.

19. Theorell T, Perski A, Akerstedt T, Sigala F, Ahlberg-Hulten G, Svensson J, Eneroth $P$ : Changes in job strain in relation to changes in physiological state. A longitudinal study Scand J Work Environ Health 1988, 14(3):189-196.

20. Karasek $R$, Choi B, Ostergren PO, Ferrario M, De Smet P: Testing two methods to create comparable scale scores between the Job Content Questionnaire (JCQ) and JCQ-like questionnaires in the European JACE Study. Int J Behav Med 2007, 14(4):189-201.

21. Coetsier P, De Backer G, De Corte W, Hellemans C, Karnas G, Kornitzer M, Pelfrenen E, Stam M, Vlerick P: Etude belge du stress au travail: Aperçu du modèle de recherche et des outils d'investigation [Belgian job stress study (BELSTRESS): overview of the study model and research methods.]. Rev Psychol Psychom 1996, 17:17-35.

22. Goldberg M, Leclerc A, Bonenfant S, Chastang JF, Schmaus A, Kaniewski N, Zins M: Cohort profile: the GAZEL Cohort Study. Int J Epidemiol 2007, 36(1):32-39. 
23. Korkeila K, Suominen S, Ahvenainen J, Ojanlatva A, Rautava P, Helenius H, Koskenvuo M: Non-response and related factors in a nation-wide health survey. Eur J Epidemiol 2001, 17(11):991-999.

24. Hasson D, Theorell T, Westerlund H, Canlon B: Prevalence and characteristics of hearing problems in a working and non-working Swedish population. J Epidemiol Community Health 2010, 64(5):453-460.

25. Magnusson Hanson LL, Theorell T, Oxenstierna G, Hyde M, Westerlund H: Demand, control and social climate as predictors of emotional exhaustion symptoms in working Swedish men and women. Scand J Public Health 2008, 36(7):737-743.

26. Alfredsson L, Hammar N, Fransson E, de Faire U, Hallqvist J, Knutsson A, Nilsson T, Theorell T, Westerholm P: Job strain and major risk factors for coronary heart disease among employed males and females in a Swedish study on work, lipids and fibrinogen. Scand J Work Environ Health 2002, 28(4):238-248.

27. Kristensen TS, Hannerz H, Hogh A, Borg V: The Copenhagen Psychosocial Questionnaire-a tool for the assessment and improvement of the psychosocial work environment. Scand J Work Environ Health 2005 31(6):438-449.

28. Burr H, Bjorner JB, Kristensen TS, Tuchsen F, Bach E: Trends in the Danish work environment in 1990-2000 and their associations with labor-force changes. Scand J Work Environ Health 2003, 29(4):270-279.

29. Vaananen A, Murray M, Koskinen A, Vahtera J, Kouvonen A, Kivimaki M: Engagement in cultural activities and cause-specific mortality: prospective cohort study. Prev Med 2009, 49(2-3):142-147.

30. Kivimaki M, Lawlor DA, Davey Smith G, Kouvonen A, Virtanen M, Elovainio M, Vahtera J: Socioeconomic position, co-occurrence of behavior-related risk factors, and coronary heart disease: the Finnish Public Sector study. Am J Public Health 2007, 97(5):874-879.

31. Stang A, Moebus S, Dragano N, Beck EM, Mohlenkamp S, Schmermund A, Siegrist J, Erbel R, Jockel KH: Baseline recruitment and analyses of nonresponse of the Heinz Nixdorf Recall Study: identifiability of phone numbers as the major determinant of response. Eur J Epidemio/ 2005, 20(6):489-496.

32. Nielsen ML, Kristensen TS, Smith-Hansen L: The Intervention Project on Absence and Well-being (IPAW): Design and results from the baseline of a 5-year study. Work Stress 2002, 16(3):191-206.

33. Holle R, Happich M, Lowel H, Wichmann HE: KORA-a research platform for population based health research. Gesundheitswesen 2005, 67(Suppl 1): S19-S25.

34. van den Bossche SNJ, Hupkens CLH, de Ree SJM, Smulders PGW: Nationale Enquête Arbeidsomstandigheden 2005: methodologie en globale resultaten. [Netherlands Working Conditions Survey, methodology and overall results. Hoofddorp: TNO Work \& Employment; 2005, 2006.

35. de Groot W, Dekker R: The Dutch System of Official Social Surveys. EuReporting Working Paper No 30 Mannheim: Mannheim Centre for European Social Research; 2001.

36. Borritz M, Rugulies R, Christensen KB, Villadsen E, Kristensen TS: Burnout as a predictor of self-reported sickness absence among human service workers: prospective findings from three year follow up of the PUMA study. Occup Environ Med 2006, 63(2):98-106.

37. Marmot MG, Smith GD, Stansfeld S, Patel C, North F, Head J, White I, Brunner E, Feeney A: Health inequalities among British civil servants: the Whitehall II study. Lancet 1991, 337(8754):1387-1393.

38. Courvoisier DS, Perneger TV: Validation of alternative formulations of job strain. [Erratum in: J Occup Health. 2010, 52(4):E4]. J Occup Health 2010, 52(1):5-13.

39. Landis JR, Koch GG: The measurement of observer agreement for categorical data. Biometrics 1977, 33(1):159-174.

40. Choi B, Bjorner JB, Ostergren PO, Clays E, Houtman I, Punnett L, Rosengren A, De Bacquer D, Ferrario M, Bilau M, et al: Cross-language differential item functioning of the job content questionnaire among European countries: the JACE study. Int J Behav Med 2009, 16(2):136-147.

41. Kivimaki M, Virtanen M, Elovainio M, Kouvonen A, Vaananen A, Vahtera J: Work stress in the etiology of coronary heart disease-a meta-analysis. Scand J Work Environ Health 2006, 32(6):431-442.

\section{Pre-publication history}

The pre-publication history for this paper can be accessed here:

http://www.biomedcentral.com/1471-2458/12/62/prepub doi:10.1186/1471-2458-12-62

Cite this article as: Fransson et al:: Comparison of alternative versions of the job demand-control scales in 17 European cohort studies: the IPDWork consortium. BMC Public Health 2012 12:62.

\section{Submit your next manuscript to BioMed Central and take full advantage of:}

- Convenient online submission

- Thorough peer review

- No space constraints or color figure charges

- Immediate publication on acceptance

- Inclusion in PubMed, CAS, Scopus and Google Scholar

- Research which is freely available for redistribution

Submit your manuscript at www.biomedcentral.com/submit 